\title{
Semiclassical fast-forward shortcuts to adiabaticity
}

\author{
Ayoti Patra 10 \\ Department of Physics, University of Maryland, College Park, Maryland 20742, USA
}

Christopher Jarzynski (i)

Department of Chemistry and Biochemistry, University of Maryland, College Park, Maryland 20742, USA; Institute for Physical Science and Technology, University of Maryland, College Park, Maryland 20742, USA; and Department of Physics, University of Maryland, College Park, Maryland 20742, USA

(Received 10 August 2020; accepted 13 January 2021; published 28 January 2021)

\begin{abstract}
In fast-forward quantum shortcuts to adiabaticity, a designed potential $U_{F F}(q, t)$ steers a wave function to evolve from the $n$th eigenstate of an initial Hamiltonian $\hat{H}(0)$ to the $n$th eigenstate of a final Hamiltonian $\hat{H}(\tau)$, in finite time $\tau$. Previously proposed strategies for constructing $U_{F F}$ are (in the absence of special symmetries) limited to the ground state $n=0$. We develop a method that overcomes this limitation, thereby substantially expanding the applicability of this shortcut to adiabaticity, and we illustrate its effectiveness with numerical simulations. Semiclassical analysis provides insight and establishes a close correspondence to the analogous classical fast-forward method.
\end{abstract}

DOI: 10.1103/PhysRevResearch.3.013087

\section{INTRODUCTION}

Shortcuts to adiabaticity (STA), i.e., strategies for attaining adiabatic outcomes in finite times, offer a set of tools for accelerating the dynamics of quantum systems, with important applications to atomic and chemical physics and quantum technologies [1-3]. Considerable theoretical [4-25] and experimental [26-32] advances have been made in this emerging field and have been extended to classical Hamiltonian [33-38] and stochastic dynamics [39-43].

For continuous systems with one degree of freedom, we formulate the problem of STA as follows (see Sec. V for a slightly different formulation). Consider a Hamiltonian

$$
\hat{H}_{0}(t)=-\frac{\hbar^{2}}{2 m} \frac{\partial^{2}}{\partial q^{2}}+U_{0}(q, t)
$$

and let $|k(t)\rangle$ or $\phi_{k}(q, t)=\langle q \mid k(t)\rangle$ denote its $k$ th eigenstate, with eigenvalue $E_{k}(t)$. Here $\phi_{k}(q, t)$ is a real function and $\hat{H}_{0}(t)$ is time dependent only in the interval $0 \leqslant t \leqslant \tau$. Initializing the system in an energy eigenstate $\psi_{t \leqslant 0}=|n(0)\rangle$, we want it to evolve to the final eigenstate $\psi_{t \geqslant \tau}=|n(\tau)\rangle$. For slow driving $(\tau \rightarrow \infty)$ the system naturally follows the adiabatic path $\psi_{t}=|n(t)\rangle$ at all times [44]. However, for rapid driving, additional measures, i.e., shortcuts, must be taken to prevent nonadiabatic transitions and guide the system to the desired final state. Here and below we suppress overall time-dependent phases when writing the state of our system.

Published by the American Physical Society under the terms of the Creative Commons Attribution 4.0 International license. Further distribution of this work must maintain attribution to the author(s) and the published article's title, journal citation, and DOI.
In one STA approach, known as fast-forward driving $[14,15,24]$, an auxiliary potential $U_{F F}(q, t)$ is added to $\hat{H}_{0}(t)$, where $U_{F F}$ is designed to guide the system to the final state $\psi_{\tau}=|n(\tau)\rangle$. This approach offers a promising tool for achieving the controlled acceleration of continuous quantum systems towards desired ends such as the manipulation of Bose-Einstein condensates [45-49] or ions in trapping potentials $[50,51]$, or population transfer between vibrational molecular states [2,52].

Unfortunately, the procedure for constructing $U_{F F}(q, t)$ generically leads to divergent behavior, specifically, infinities in the potential, at locations where the eigenstate $\phi_{n}(q, t)$ has nodes $[1,12,42]$. As a result, this method has been restricted to ground-state wave functions, which lack nodes, or to situations in which special scale-invariant symmetries eliminate the divergences [7]. Truncating divergences with finite cutoffs has proven useful when driving a system from its ground state to its first excited state [i.e., $|0(0)\rangle \rightarrow|1(\tau)\rangle][12]$, but this strategy has not been explored for the STA problem formulated above $[|n(0)\rangle \rightarrow|n(\tau)\rangle]$ and it is likely to lead to difficult-to-engineer potentials for eigenstates with many nodes.

In this paper we extend the fast-forward approach to excited states, by developing a semiclassical strategy that is free of divergences. In Sec. II we derive our main result: a semiclassically motivated recipe for constructing a divergence-free $U_{F F}(q, t)$ for excited energy eigenstates. In Sec. III we use numerical simulations to illustrate the effectiveness of this approach. We find that our fast-forward potential steers the wave function to the desired final eigenstate with high accuracy, with only a small amount of probability spilling out into sideband states. In Sec. IV we compare these results with corresponding classical simulations and we develop a semiclassical theory for these sidebands. We end with 
a brief discussion in Sec. V. Several technical steps of our analysis are relegated to the Appendix, for clarity of presentation.

\section{DERIVATION OF THE MAIN RESULT}

As in Refs. [35,42], our starting point is a velocity field $v(q, t)$ (as yet undetermined) that vanishes for $t \notin[0, \tau]$. We then use $v(q, t)$ to define an acceleration field $a(q, t)$, from which the fast-forward potential is constructed, as follows:

$$
\partial_{q} U_{F F}(q, t)=-m a(q, t) \equiv-m\left(v \partial_{q} v+\partial_{t} v\right) .
$$

Given $v(q, t)$, Eq. (2) defines $U_{F F}(q, t)$ up to an integration constant $u_{F F}(t)$, which is chosen so that $U_{F F}=0$ for $t \notin$ $[0, \tau]$. Thus $v, a$, and $U_{F F}$ all vanish outside the interval $[0, \tau]$.

The task now is to design $v(q, t)$ so that $U_{F F}(q, t)$ [given by Eq. (2)] generates the desired shortcut. To this end, we define a function $S(q, t)$ via the relation

$$
\partial_{q} S(q, t)=m v(q, t)
$$

where the integration constant $s(t)$ is adjusted (see the Appendix for details) so that $S$ obeys the Hamilton-Jacobi equation

$$
\frac{\partial S}{\partial t}+\frac{1}{2 m}\left(\frac{\partial S}{\partial q}\right)^{2}+U_{F F}=0 .
$$

By Eq. (3), $S(q, t)=S_{-}$for $t \leqslant 0$ and $S(q, t)=S_{+}$for $t \geqslant \tau$, for some constants $S_{ \pm}$.

With these definitions in place, we propose the ansatz

$$
\bar{\psi}(q, t)=\phi_{n}(q, t) e^{i S(q, t) / \hbar} \exp \left[-\frac{i}{\hbar} \int_{0}^{t} E_{n}\left(t^{\prime}\right) d t^{\prime}\right]
$$

(for a given $n \geqslant 0$ ) as a solution to the time-dependent Schrödinger equation (TDSE)

$$
i \hbar \frac{\partial \bar{\psi}}{\partial t}=\left(\hat{H}_{0}+\hat{U}_{F F}\right) \bar{\psi} .
$$

Note that $\bar{\psi}_{t \leqslant 0}=|n(0)\rangle$ and $\bar{\psi}_{t \geqslant \tau}=|n(\tau)\rangle$. Substituting Eq. (5) into Eq. (6) yields (see the Appendix)

$$
i \hbar \frac{\partial \phi_{n}}{\partial t}=\frac{1}{2}(\hat{p} \hat{v}+\hat{v} \hat{p}) \phi_{n} \equiv \hat{D}(t) \phi_{n},
$$

where $\hat{p}=-i \hbar \partial_{q}$ and $\hat{v}(t)=v(\hat{q}, t)$. Thus, if we can find a function $v(q, t)$ that satisfies Eq. (7), then by construction our ansatz $\bar{\psi}$ [Eq. (5)] will satisfy the TDSE and will have the desired property of beginning and ending in the $n$th energy eigenstate. In other words, our ansatz converts the problem of constructing a potential $U_{F F}$ that satisfies Eq. (6) to that of constructing a velocity field $v$ that satisfies Eq. (7) for a given choice of $n$. We now explore strategies for accomplishing this task. In what follows, we drop the subscript $n$ and simply write $\phi(q, t)$ and $E(t)$ for the $n$th eigenstate and eigenenergy of $\hat{H}_{0}(t)$, respectively.

Equation (7) implies (see the Appendix)

$$
\partial_{t} \phi^{2}+\partial_{q}\left(v \phi^{2}\right)=0,
$$

which is the continuity equation for a probability density $\phi^{2}(q, t)$ evolving under the deterministic flow $d q / d t=$ $v(q, t)$. Thus it seems we should construct $v(q, t)$ to satisfy
Eq. (8). Unfortunately, the resulting velocity field generically diverges at the nodes of the eigenstate, where $\phi^{2}=0$ [42]. In fact, constructing $U_{F F}$ via Eq. (8) is equivalent to previously proposed fast-forward approaches $[14,15,24]$ and leads to the divergent behavior mentioned earlier. Let us therefore try a different strategy, motivated by semiclassical arguments.

In the WKB approximation, an excited eigenstate of $\hat{H}_{0}(t)$ is given by [44]

$$
\phi(q, t)=\sqrt{\frac{\rho}{2}}\left(e^{i \Sigma / \hbar-i \pi / 4}+e^{-i \Sigma / \hbar+i \pi / 4}\right) \equiv \phi_{+}+\phi_{-},
$$

where

$$
\begin{aligned}
& \Sigma(q, t)=\int_{q_{L}}^{q} \bar{p}\left(q^{\prime}, t\right) d q^{\prime}, \quad \bar{p}=\sqrt{2 m\left(E-U_{0}\right)}, \\
& \rho(q, t) \propto \frac{1}{\bar{p}} .
\end{aligned}
$$

Here we treat $t$ as a fixed parameter and we consider a classical trajectory of energy $E=E(t)$ evolving under the Hamiltonian $H_{0}=p^{2} / 2 m+U_{0}(q, t)$. Then $q_{L}(t)$ is the left turning point of the trajectory, $\pm \bar{p}(q, t)$ are the momenta on the upper $(+)$ and lower $(-)$ branches of the energy shell (i.e., level set) $H_{0}(q, p, t)=E(t)$, and $\rho(q, t)$ is the position probability density sampled by the trajectory over one period of oscillation. In Eq. (10) and below, we replace the eigenenergy $E$ by its WKB approximation, determined by $\oint_{E} \bar{p} d q=2 \pi \hbar\left(n+\frac{1}{2}\right)$.

The eigenstate probability distribution $\phi^{2}(q, t)$ oscillates with $q$ due to interference between $\phi_{+}$and $\phi_{-}$[Eq. (9)], but after local averaging over these oscillations, $\phi^{2}(q, t)$ is semiclassically equivalent to $\rho(q, t)$ [44]. Since the troublesome nodes in $\phi$ arise from destructive interference between $\phi_{+}$and $\phi_{-}$, let us see what happens if we attempt to construct $v(q, t)$ to satisfy Eq. (7) separately for each term: $i \hbar \partial_{t} \phi_{ \pm}=\hat{D} \phi_{ \pm}$. Upon substitution and separation of real and imaginary parts (see Appendix) we obtain

$$
\begin{aligned}
& \partial_{t} \Sigma+v \partial_{q} \Sigma=0, \\
& \partial_{t} \rho+\partial_{q}(v \rho)=0 .
\end{aligned}
$$

To interpret these conditions, we observe that $\phi_{ \pm}(q, t)$ reflects both a classical probability distribution $\rho(q, t)$ and a quantum phase $e^{ \pm i(\Sigma / \hbar-\pi / 4)}$. Equation (11b) is a continuity equation for $\rho(q, t)$ under the flow $d q / d t=v(q, t)$, whereas Eq. (11a) implies that the phase remains constant, $d \Sigma / d t=0$, under this flow. By Eq. (9) the nodes $\left\{q_{v}(t)\right\}$ of $\phi(q, t)$ satisfy

$$
\Sigma\left(q_{v}(t), t\right)=\left(v-\frac{1}{4}\right) \pi \hbar, \quad 1 \leqslant v \leqslant n
$$

thus the value of $\Sigma$ remains constant along a node $q_{v}(t)$, as the parameter $t$ is varied. Hence Eq. (11a) implies that the field $v(q, t)$ describes the nodal velocities (with respect to $t$ ), as illustrated in Fig. 1.

Equations (11a) and (11b) are not generally satisfied by a single $v(q, t)$. We have thus replaced one condition, Eq. (8), 


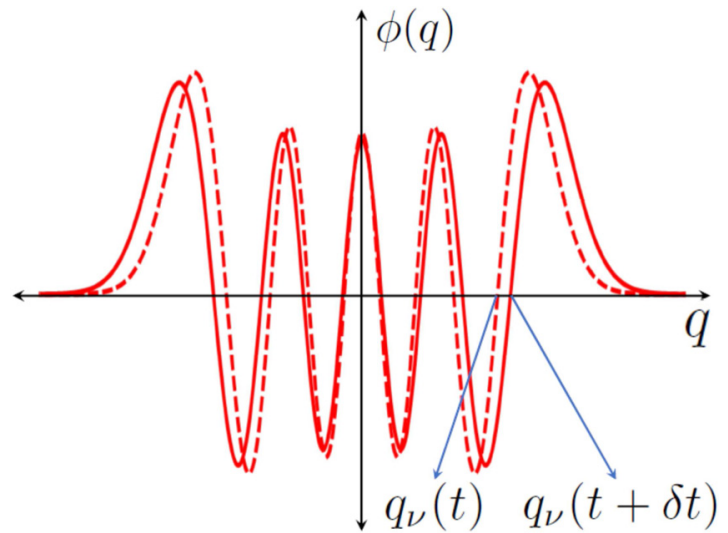

FIG. 1. Energy eigenstate $\phi$ at times $t$ and $t+\delta t$ (dashed and solid curves). By Eq. (11a), the node $q_{v}(t)$ is displaced by $v\left(q_{v}, t\right) d t$ when $t \rightarrow t+d t$.

that suffers from divergences, with a pair of conditions, Eqs. (11), that cannot be simultaneously satisfied. To make further progress, we choose to satisfy Eq. (11a) rather than Eq. (11b). Thus we take

$$
v(q, t)=-\frac{\partial_{t} \Sigma}{\partial_{q} \Sigma}
$$

and construct $U_{F F}$ using Eq. (2), in the hope that this fastforward potential will accurately steer the wave function to the desired final adiabatic state. A posteriori justification of this choice will be provided later. First we describe a test of this approach, using numerical simulations of a model system evolving under the time-dependent Schrödinger equation.

\section{NUMERICAL RESULTS}

We simulated a quantum particle of unit mass evolving in a potential

$$
U_{0}(q, t)=q^{4}-16 q^{2}+\lambda(t) q,
$$

where $\lambda$ varies from +16 to -16 as $\lambda(t)=4 \cos (\pi t / \tau)[5-$ $\cos (2 \pi t / \tau)]$, with $\tau=1.0$. The system was initialized in the state $\psi_{0}=|n(0)\rangle$ and then evolved under the time-dependent Schrödinger equation, first using the Hamiltonian $\hat{H}_{0}(t)$ and then using $\hat{H}_{0}(t)+\hat{U}_{F F}(t)$, with the fast-forward potential determined by Eqs. (2) and (13) as described above.

Figure 2 presents numerical results for $n=17$, setting $\hbar=2$. Each frame displays a histogram showing how the evolving wave function $\psi_{t}$ is distributed among the instantaneous eigenstates $\left|\phi_{k}(t)\right\rangle$, as quantified by the overlap $p_{k}(t)=$ $\left|\left\langle\phi_{k} \mid \psi_{t}\right\rangle\right|^{2}$. Figures 2(a)-2(c) and 2(d)-2(f) correspond to evolution under $\hat{H}_{0}$ and $\hat{H}_{0}+\hat{U}_{F F}$, respectively. In both cases the system evolves from $\psi_{0}=|17\rangle$ [Figs. 2(a) and 2(d)] to a superposition of energy eigenstates at intermediate times [Figs. 2(b) and 2(e)]. While the system evolving under $\hat{H}_{0}(t)$ ends in a broad superposition of eigenstates [Fig. 2(c)], the one evolving with the fast-forward shortcut reaches the final adiabatic state to a very good approximation: In Fig. 2(f), $p_{17}=0.91$ and $p_{16}+p_{17}+p_{18}=0.98$. Thus the potential $U_{F F}(q, t)$ constructed from Eqs. (2) and (13) achieves fastforward driving with high accuracy. We have performed simulations using different parameter values and have found that the potential $\hat{U}_{F F}$ consistently guides the system to a final state whose probability is concentrated in the target eigenstate $|n(\tau)\rangle$, along with a few sideband states such as $k=16,18$ in Fig. 2(f). Animations of further simulations are found in Ref. [53].
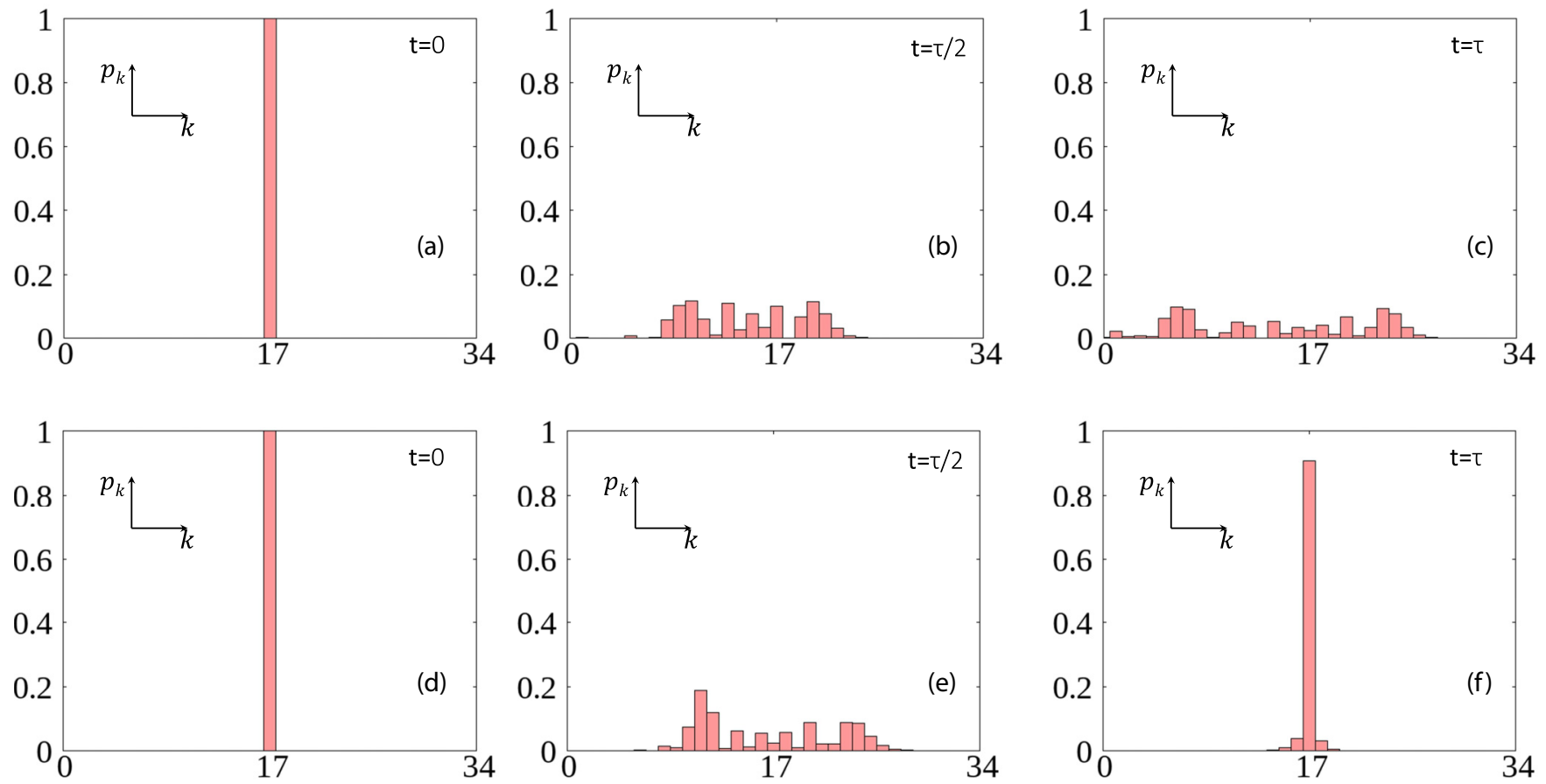

FIG. 2. Overlap $p_{k}(t)=\left|\left\langle\phi_{k}(t) \mid \psi(t)\right\rangle\right|^{2}$ between the eigenstates of $\hat{H}_{0}(t)$ and the wave function $\psi(t)$, as it evolves under (a)-(c) $\hat{H}_{0}(t)$ or (d)-(f) $\hat{H}_{0}(t)+\hat{U}_{F F}(t)$. 


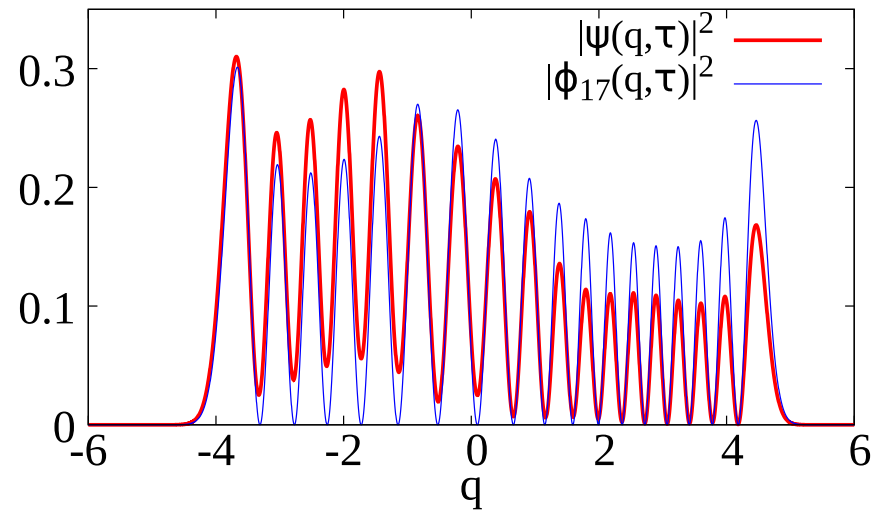

FIG. 3. Final probability distribution $|\psi(q, \tau)|^{2}$ (thick red line) and eigenstate distribution $\left|\phi_{17}(q, \tau)\right|^{2}$ (thin blue line) plotted for evolution under $\hat{H}_{0}+\hat{U}_{F F}$ from the initial state $\psi(q, 0)=\phi_{17}(q, 0)$.

For evolution under $\hat{H}_{0}+\hat{U}_{F F}$, Fig. 3 shows the distributions $|\psi(q, \tau)|^{2}$ and $\left|\phi_{17}(q, \tau)\right|^{2}$. While the minima and maxima align nicely, the values of $|\psi|^{2}$ and $\left|\phi_{17}\right|^{2}$ differ visibly. This is understandable, as $v(q, t)$ was constructed to track the eigenstate's phase $\Sigma$ [Eq. (11a)] rather than its magnitude $\rho$ [Eq. (11b)]. The mismatch in Fig. 3 is reflected in the sidebands in Fig. 2(f). We now develop intuition by comparing our results with classical simulations and we derive a semiclassical prediction for the sidebands [Eq. (15)].

\section{CLASSICAL RESULTS AND SEMICLASSICAL ANALYSIS}

We first note that the potential $U_{F F}(q, t)$ determined by Eqs. (2) and (13) is identical to the one designed in Ref. [35] [see Eqs. (8)-(12) therein] for a purely classical fast-forward shortcut. This agreement reflects the correspondence principle (CP) and provides a measure of justification for choosing to satisfy Eq. (11a) rather than Eq. (11b). Indeed, by invoking the CP we could have argued for constructing $U_{F F}(q, t)$ from Eqs. (2) and (13) directly from the classical results of Ref. [35], rather than by proceeding via the WKB approximation as done above. These considerations motivate us to compare our quantum simulations with classical counterparts.

Figure 4 depicts snapshots of 50 trajectories evolving under the classical counterpart of the Hamiltonian $\hat{H}_{0}+\hat{U}_{F F}$ used to generate Figs. 2(d)-2(f) above, with $\tau=1.0$. Initial conditions were spaced uniformly, with respect to the angle $\theta$ of action-angle coordinates $(I, \theta)$ [54], over an energy shell
$H_{0}(q, p, 0)=53.86=E_{17}(0)$. The closed black curves depict an energy shell $\mathcal{E}(t)$ determined by a fixed value of the action $I=(2 \pi)^{-1} \oint \bar{p} d q$. We refer to $\mathcal{E}(t)$ as the adiabatic energy shell.

The classical action $I$ is an adiabatic invariant: For slow driving $(\tau \rightarrow \infty)$, trajectories evolving under $H_{0}(q, p, t)$ cling to the adiabatic shell $\mathcal{E}(t)$ at all times, whereas for rapid driving $(\tau=1.0)$ the trajectories evolve away from the adiabatic shell [35]. Figure 4 illustrates the effect of adding the fastforward potential $U_{F F}$ when $\tau=1.0$ : The trajectories at first stray off shell [Fig. 4(b)], but return faithfully to the adiabatic shell at $t=\tau$ [Fig. 4(c)].

Figures 4(a)-4(c) are classical counterparts of Figs. 2(d)2(f). Observe that both final states display "imperfections": The wave function in Fig. 2(f) does not end exactly in the eigenstate $\phi_{17}(\tau)$ and the trajectories in Fig. 4(c) are distributed nonuniformly over the energy shell $\mathcal{E}(\tau)$. These features are related. Using time-dependent WKB theory [55], we obtain the following prediction for the overlap between the final wave function $\psi(q, \tau)$ and the eigenstate $\phi_{n+l}(q, \tau)$ (see Appendix):

$$
\left|\left\langle\phi_{n+l} \mid \psi\right\rangle\right|_{t=\tau}^{2}=\left|\int_{0}^{2 \pi} d \theta e^{-i l \theta} \sqrt{\frac{\eta(\theta, \tau)}{2 \pi}}\right|^{2},
$$

where $\eta$ is the classical distribution of final conditions on the energy shell $\mathcal{E}(\tau)$, expressed in the angle variable $\theta$. Equation (15) relates the sidebands in Fig. 2(f) to the nonuniformity of points in Fig. 4(c); if the classical distribution were uniform $\eta=1 / 2 \pi$, the sidebands would vanish: $\left|\left\langle\phi_{n+l} \mid \psi\right\rangle\right|_{t=\tau}^{2}=\delta_{l 0}$.

Figure 5 compares the histogram appearing in Fig. 2(f) with the prediction of Eq. (15), evaluated using the trajectories in Fig. 4(c). The evident agreement supports our semiclassical analysis, which suggests that the nonzero sidebands will persist even in the limit $\hbar \rightarrow 0$. Namely, imagine that for the same $\hat{H}_{0}(t)$ used to generate Fig. 2 we tuned the value of $\hbar$ (keeping other parameters unchanged) so that $E=53.86$ were the 1017 th, rather than 17 th, eigenenergy of $\hat{H}(0)$ and imagine we then reran both the quantum and classical simulations. Since the latter are unaffected by the choice of $\hbar$, we would again obtain Fig. 4, but the quantum simulations would produce a final histogram $p_{k}(\tau)$ sharply peaked at $k=$ 1017. Equation (15) implies that the distribution of probability among the eigenstates $k=1014-1020$ would be the same as that observed among $k=14-20$ in Figs. 2(f) and 5. This implies that in the limit $\hbar \rightarrow 0$, the variance of the energy distribution associated with the nonzero sidebands tends to zero (since the energy level spacing tends to zero), consistent
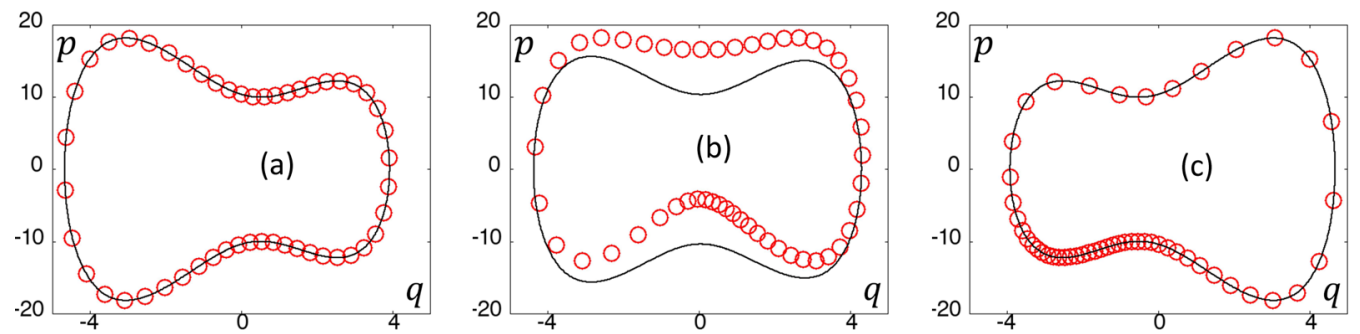

FIG. 4. Snapshots of trajectories evolving in phase space under $H_{0}(q, p, t)+U_{F F}(q, t)$, the classical counterpart of the Hamiltonian used to generate Figs. 2(d) $-2(\mathrm{f})$, for (a) $t=0$, (b) $t=\tau / 2$, and (c) $t=\tau$. The closed black curves show the adiabatic energy shell $\mathcal{E}(t)$ of $H_{0}(q, p, t)$. 


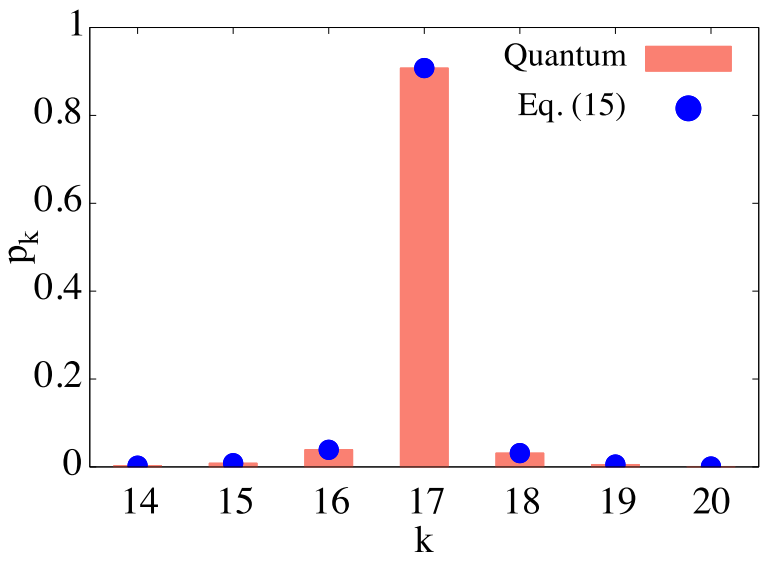

FIG. 5. Comparison between $p_{k}=\left|\left\langle\phi_{k} \mid \psi\right\rangle\right|^{2}$ (red bars), obtained from quantum evolution under $\hat{H}_{0}+\hat{U}_{F F}$, and the prediction of Eq. (15) (blue circles), evaluated using the classical trajectories of Fig. 4.

with a classical final state in which all trajectories end on the adiabatic energy shell [Fig. 4(c)].

\section{CONCLUSION}

We have expanded the toolkit for accelerating quantum dynamics, by developing a method for constructing fast-forward shortcuts that are free of divergences and thereby applicable to excited states of generic kinetic-plus-potential Hamiltonians in one degree of freedom. Our approach is the semiclassical analog of the classical method of Ref. [35]. It will be interesting to extend our approach to systems with $d>1$ degrees of freedom. For integrable systems the existence of WKB expressions for energy eigenstates is likely to prove useful to this end, but for systems with chaotic classical dynamics the task may prove challenging. As noted elsewhere, in a number of experimentally relevant situations a separation of variables may effectively reduce a $d=3$ system to a $d=1$ system [35]. Finally, while we have chosen to satisfy Eq. (11a) in our construction of $U_{F F}(q, t)$ and have used the correspondence principle to justify our choice, it will be interesting to test numerically whether satisfying Eq. (11b) instead would lead to a fast-forward potential capable of steering the wave function (approximately) to the desired final energy eigenstate.

From the start, we have assumed that the Hamiltonian $\hat{H}_{0}(t)=\hat{p}^{2} / 2 m+U_{0}(\hat{q}, t)$ is specified for all $t \in[0, \tau]$. However, in other formulations of shortcuts to adiabaticity only initial and final Hamiltonians $\hat{H}_{0}(0)$ and $\hat{H}_{0}(\tau)$ are given and the problem is then to construct a Hamiltonian $\hat{H}(t)$ under which an initial state $\psi(q, 0)=\phi_{n}(q, 0)$ evolves to a final state $\psi(q, \tau)=\phi_{n}(q, \tau)$. In this situation, we can simply choose a potential $U_{0}(q, t)$ that interpolates smoothly from $U_{0}(q, 0)$ to $U_{0}(q, \tau)$ and then apply our method to obtain $U_{F F}(q, t)$. The Hamiltonian $\hat{H}(t)=\hat{p}^{2} / 2 m+U_{0}(\hat{q}, t)+$ $U_{F F}(\hat{q}, t)$ then generates the desired evolution.

It is instructive to compare our results with those obtained for scale-invariant Hamiltonians [7], which are characterized by time dependence of the form

$$
\hat{H}_{0}(t)=\frac{\hat{p}^{2}}{2 m}+\frac{1}{\gamma^{2}} U_{0}\left(\frac{\hat{q}-f}{\gamma}\right), \quad f=f(t), \gamma=\gamma(t) .
$$

Here the parameters $f(t)$ and $\gamma(t)$ describe translations and dilations of the potential energy function. ${ }^{1}$ For Hamiltonians $\hat{H}_{0}(t)$ of this form, Eqs. (8), (11a), and (11b) are exactly satisfied by the nondivergent velocity field $v(q, t)=\dot{f}-$ $(\dot{\gamma} / \gamma)(q-f)$, where overdots indicate derivatives with respect to time. Equation (2) then leads to the fast-forward potential

$$
U_{F F}(q, t)=-m \ddot{f} q-\frac{m}{2} \frac{\ddot{\gamma}}{\gamma}(q-f)^{2} .
$$

The same potential was obtained in Ref. [7] using a trick involving canonical or unitary transformations of variables. This comparison suggests that the success of that trick may be related to the fact that Eqs. (8), (11a), and (11b) are solved by a single well-behaved field $v(q, t)$, which is a particular feature of scale-invariant Hamiltonians. If this is the case, then the approach of Ref. [7] might not readily generalize beyond scale-invariant Hamiltonians. This issue deserves further investigation.

\section{ACKNOWLEDGMENTS}

We gratefully acknowledge financial support from the U.S. Army Research Office under Contract No. W911NF-13-10390 (A.P.) and the U.S. National Science Foundation under Grant No. DMR-1506969 (C.J.).

\section{APPENDIX}

Here we provide technical details of the derivations of Eqs. (4), (7), (8), (11), and (15).

\section{Derivation of Eq. (4)}

Suppose we have constructed a function $S^{\circ}(q, t)$ that satisfies $\partial_{q} S^{\circ}=m v$ [Eq. (3)], without as yet adjusting the constant of integration $s(t)$. From Eqs. (2) and (3) we obtain

$$
\begin{aligned}
\partial_{t} \partial_{q} S^{\circ} & =m \partial_{t} v=m\left(a-v \partial_{q} v\right) \\
& =-\partial_{q}\left(U_{F F}+\frac{m v^{2}}{2}\right)
\end{aligned}
$$

or equivalently

$$
\partial_{q}\left[\partial_{t} S^{\circ}+\frac{\left(\partial_{q} S^{\circ}\right)^{2}}{2 m}+U_{F F}\right]=0 ;
$$

hence

$$
\partial_{t} S^{\circ}+\frac{\left(\partial_{q} S^{\circ}\right)^{2}}{2 m}+U_{F F}=\beta(t)
$$

\footnotetext{
${ }^{1}$ We note in passing that a harmonic oscillator described by the potential $k(t)[q-f(t)]^{2} / 2$ falls into this class, with $U_{0}(x)=x^{2} / 2$ and $\gamma=k^{-1 / 4}$.
} 
for some $\beta(t)$. By setting

$$
S(q, t)=S^{\circ}(q, t)-\int_{0}^{t} \beta\left(t^{\prime}\right) d t^{\prime}
$$

we arrive at an $S(q, t)$ that satisfies both Eqs. (3) and (4).
2. Derivation of Eq. (7)

Substituting $\psi(q, t)$ given by Eq. (5) into the timedependent Schrödinger equation

$$
i \hbar \frac{\partial \psi}{\partial t}=\left(\hat{H}_{0}+\hat{U}_{F F}\right) \psi,
$$

we separately evaluate each side using Eqs. (1) and (4) to obtain (dropping the subscript $n$ )

$$
\begin{aligned}
i \hbar \frac{\partial \psi}{\partial t} & =(i \hbar \dot{\phi}-\dot{S} \phi+E \phi) e^{i S / \hbar} e^{i \gamma}=\left[i \hbar \dot{\phi}+\left(\frac{S^{\prime 2}}{2 m}+U_{F F}+E\right) \phi\right] e^{i S / \hbar} e^{i \gamma}, \\
\left(\hat{H}_{0}+\hat{U}_{F F}\right) \psi & =\left[-\frac{\hbar^{2}}{2 m} \phi^{\prime \prime}-\frac{i \hbar}{2 m}\left(S^{\prime \prime} \phi+2 S^{\prime} \phi^{\prime}\right)+\left(\frac{S^{\prime 2}}{2 m}+U_{0}+U_{F F}\right) \phi\right] e^{i S / \hbar} e^{i \gamma},
\end{aligned}
$$

where overdots and primes denote $\partial_{t}$ and $\partial_{q}$, respectively, and $\gamma(t)=-(1 / \hbar) \int_{0}^{t} E\left(t^{\prime}\right) d t^{\prime}$. Setting the expressions in (A6) equal to one another and using $\hat{H}_{0} \phi=E \phi$, we arrive at

$$
i \hbar \dot{\phi}=-\frac{i \hbar}{2 m}\left(S^{\prime \prime} \phi+2 S^{\prime} \phi^{\prime}\right) .
$$

Using Eq. (3), we then get

$$
\begin{aligned}
i \hbar \dot{\phi} & =-\frac{i \hbar}{2}\left(v^{\prime} \phi+2 v \phi^{\prime}\right)=-\frac{i \hbar}{2}\left[\partial_{q}(v \phi)+v \partial_{q} \phi\right] \\
& =\frac{1}{2}(\hat{p} \hat{v}+\hat{v} \hat{p}) \phi,
\end{aligned}
$$

which is the desired result [Eq. (7)].

\section{Derivation of Eq. (8)}

Dividing both sides of Eq. (7) by $i \hbar$ and using $\hat{p}=-i \hbar \partial_{q}$, we get

$$
\frac{\partial \phi}{\partial t}=-\frac{1}{2 \phi} \frac{\partial}{\partial q}\left(v \phi^{2}\right) .
$$

Multiplying both sides by $2 \phi$, we obtain

$$
\frac{\partial \phi^{2}}{\partial t}=-\frac{\partial}{\partial q}\left(v \phi^{2}\right)
$$

which is the continuity equation for the probability density $\phi^{2}(q, t)$.

\section{Derivation of Eq. (11)}

Writing

$$
\phi_{+}(q, t)=\frac{\alpha}{\sqrt{2}} A e^{i \Sigma / \hbar},
$$

where $A=\sqrt{\rho}$ and $\alpha=e^{-i \pi / 4}$, and substituting this expression into $i \hbar \partial_{t} \phi_{+}=\hat{D} \phi_{+}$, the two sides evaluate as follows (dropping the extraneous factor $\alpha / \sqrt{2}$ ):

$$
\begin{aligned}
i \hbar \frac{\partial}{\partial t}\left(A e^{i \Sigma / \hbar}\right) & =(i \hbar \dot{A}-\dot{\Sigma} A) e^{i \Sigma / \hbar}, \\
\hat{D}\left(A e^{i \Sigma / \hbar}\right) & =-\frac{i \hbar}{2}\left(v^{\prime} A+2 v A^{\prime}+2 v A \frac{i}{\hbar} \Sigma^{\prime}\right) e^{i \Sigma / \hbar} .
\end{aligned}
$$

Equating these two expressions, dividing both sides of the equation by $e^{i \Sigma / \hbar}$, and then collecting real and imaginary terms, we obtain

$$
-\dot{\Sigma} A=v A \Sigma^{\prime}, \quad \dot{A}=-\frac{1}{2} v^{\prime} A-v A^{\prime} .
$$

Dividing the first equation by $A$ and multiplying the second by $2 A$, we arrive at Eq. (11). Evaluating $i \hbar \partial_{t} \phi_{-}=\hat{D} \phi_{-}$produces identical results.

\section{Derivation of Eq. (15)}

Time-dependent WKB theory [55] provides a set of tools for constructing approximations to quantum wave functions evolving under the Schrödinger equation, from ensembles of classical trajectories obeying Hamiltonian dynamics. Applying these tools to the final wave function $\psi(q, \tau)$ after evolution under $\hat{H}_{0}+\hat{U}_{F F}$, we obtain (aside from an overall phase that we ignore)

$$
\psi(q, \tau)=\sqrt{\eta_{+}} \alpha e^{i \Sigma_{n} / \hbar}+\sqrt{\eta_{-}} \alpha^{*} e^{-i \Sigma_{n} / \hbar},
$$

where $\alpha=e^{-i \pi / 4}, \eta_{ \pm}(q, \tau)$ denote the classical probability distributions on the upper and lower branches of the energy shell $E=E_{n}(\tau)$, depicted in Fig. 4(c), and $\Sigma_{n}(q, t)$ is given by Eq. (10). At a given location $q$, the two terms appearing on the right-hand side of Eq. (A14) describe a right-moving and a left-moving wave train, with local momenta $\pm \bar{p}= \pm \partial_{q} \Sigma_{n}$ (since the classical trajectories all end on the adiabatic energy shell) and amplitudes $\sqrt{\eta_{ \pm}}$.

We wish to take the inner product between $\psi(q, \tau)$ and the $k$ th energy eigenstate $\phi_{k}(q, \tau)$ for $k \approx n$. Time-dependent WKB theory gives [see also Eq. (9)]

$$
\phi_{k}(q, \tau)=\sqrt{\rho_{k+}} \alpha e^{i \Sigma_{k} / \hbar}+\sqrt{\rho_{k-}} \alpha^{*} e^{-i \Sigma_{k} / \hbar},
$$

where $\rho_{k \pm}=\rho_{k} / 2$ and the subscript $k$ indicates that we are evaluating $\Sigma(q, \tau)$ and $\rho(q, \tau)$ [Eq. (10)] at the energy $E=$ $E_{k}(\tau)$. The functions $\rho_{k \pm}$ represent equal contributions to the probability distribution $\rho_{k}$ from the two branches of the energy shell. 
Taking the inner product between $\phi_{k}$ and $\psi$ gives the contribution

$$
\begin{aligned}
\left\langle\phi_{k} \mid \psi\right\rangle_{+} & =\int_{q_{L}}^{q_{R}} d q \sqrt{\rho_{k+} \eta_{+}} e^{i\left(\Sigma_{n}-\Sigma_{k}\right) / \hbar} \\
& =\int_{q_{L}}^{q_{R}} \rho_{k+} d q \sqrt{\frac{\eta_{+}}{\rho_{k+}}} e^{i\left(\Sigma_{n}-\Sigma_{k}\right) / \hbar}
\end{aligned}
$$

from the upper branch and a similar contribution $\left\langle\phi_{k} \mid \psi\right\rangle_{-}$ from the lower branch. (We discard cross-term contributions between the two branches, as they approximately vanish upon integrating over rapidly oscillating phases.) We now use action-angle coordinates $(\theta, I)$ to analyze these contributions. In what follows, we mostly suppress the dependence of various quantities on the constant $\tau$.

The action $I$ corresponding to energy $E$ is given by

$$
I(E)=\frac{1}{2 \pi} \oint_{E} \bar{p} d q
$$

Since the function $\Sigma$ is evaluated on a particular energy shell $E$, it can be viewed as a function of the action $\Sigma=\Sigma(q, I)$, where $I=I(E)$. Written in this way, it is the generating function for a canonical transformation from coordinates $(q, p)$ to $(\theta, I)$ [54]. On the upper branch of the energy shell, the angle variable $\theta \in[0, \pi]$ is given by

$$
\theta(q, I)=\frac{\partial \Sigma}{\partial I} .
$$

Defining $I_{j} \equiv I\left(E_{j}\right)=\hbar\left(j+\frac{1}{2}\right)$, we obtain

$$
\begin{aligned}
\Sigma_{n}(q)-\Sigma_{k}(q) & =\Sigma\left(q, I_{n}\right)-\Sigma\left(q, I_{k}\right) \approx \frac{\partial \Sigma}{\partial I}\left(I_{n}-I_{k}\right) \\
& =\theta \hbar(n-k) .
\end{aligned}
$$

The distribution $\rho_{k+}(q)$ is uniform in the angle variable $\rho_{k+}(q) d q=d \theta / 2 \pi$, which allows us to perform a change of variables, from $q$ to $\theta$, in the evaluation of Eq. (A16),

$\left\langle\phi_{k} \mid \psi\right\rangle_{+}=\int_{0}^{\pi} \frac{d \theta}{2 \pi} \sqrt{\frac{2 \pi \eta_{+}}{|d \theta / d q|}} e^{i(n-k) \theta}=\int_{0}^{\pi} d \theta \sqrt{\frac{\eta}{2 \pi}} e^{i(n-k) \theta}$,

where

$$
\eta(\theta, \tau)=\eta_{+}(q, \tau)\left|\frac{\partial q}{\partial \theta}\right|, \quad 0<\theta<\pi
$$

is the probability distribution on the upper branch of the energy shell, given in terms of the angle variable $\theta$. For the lower branch of the energy shell we have $\theta=-\partial \Sigma / \partial I$ and $\rho_{k-} d q=-d \theta / 2 \pi$ and we obtain

$$
\begin{aligned}
\left\langle\phi_{k} \mid \psi\right\rangle_{-} & =\int_{q_{L}}^{q_{R}} \rho_{k-} d q \sqrt{\frac{\eta_{-}}{\rho_{k-}}} e^{-i\left(\Sigma_{n}-\Sigma_{k}\right) / \hbar} \\
& =\int_{-\pi}^{0} d \theta \sqrt{\frac{\eta}{2 \pi}} e^{i(n-k) \theta},
\end{aligned}
$$

with

$$
\eta(\theta, \tau)=\eta_{-}(q, \tau)\left|\frac{\partial q}{\partial \theta}\right|, \quad-\pi<\theta<0 .
$$

Summing the two contributions, we get

$$
\left\langle\phi_{k} \mid \psi\right\rangle=\int_{-\pi}^{\pi} d \theta \sqrt{\frac{\eta(\theta, \tau)}{2 \pi}} e^{i(n-k) \theta} .
$$

Replacing $\int_{-\pi}^{\pi} d \theta$ with $\int_{0}^{2 \pi} d \theta$ and setting $l=k-n$, we arrive at Eq. (15).
[1] D. Guéry-Odelin, A. Ruschhaupt, A. Kiely, E. Torrontegui, S. Martínez-Garaot, and J. G. Muga, Shortcuts to adiabaticity: Concepts, methods, and applications, Rev. Mod. Phys. 91, 045001 (2019).

[2] S. Masuda and S. A. Rice, Controlling quantum dynamics with assisted adiabatic processes, Adv. Chem. Phys. 159, 51 (2016).

[3] E. Torrontegui, S. Ibáñez, S. Martínez-Garaot, M. Modugno, A. del Campo, D. Guéry-Odelin, A. Ruschhaupt, X. Chen, and J. G. Muga, Shortcuts to adiabaticity, Adv. At. Mol. Opt. Phys. 62, 117 (2013).

[4] M. V. Berry, Transitionless quantum driving, J. Phys. A: Math. Theor. 42, 365303 (2009).

[5] X. Chen, E. Torrontegui, and J. G. Muga, Lewis-Riesenfeld invariants and transitionless quantum driving, Phys. Rev. A 83, 062116 (2011).

[6] S. Deffner, Shortcuts to adiabaticity: Suppression of pair production in driven Dirac dynamics, New J. Phys. 18, 012001 (2016).

[7] S. Deffner, C. Jarzynski, and A. del Campo, Classical and Quantum Shortcuts to Adiabaticity for Scale-Invariant Driving, Phys. Rev. X 4, 021013 (2014).

[8] A. del Campo, Shortcuts to Adiabaticity by Counterdiabatic Driving, Phys. Rev. Lett. 111, 100502 (2013).
[9] M. Demirplak and S. A. Rice, Assisted adiabatic passage revisited, J. Phys. Chem. B 109, 6838 (2005).

[10] S. Ibáñez, X. Chen, E. Torrontegui, J. G. Muga, and A. Ruschhaupt, Multiple Schrödinger Pictures and Dynamics in Shortcuts to Adiabaticity, Phys. Rev. Lett. 109, 100403 (2012).

[11] S. Ibáñez, S. Martínez-Garaot, X. Chen, E. Torrontegui, and J. G. Muga, Shortcuts to adiabaticity for non-Hermitian systems, Phys. Rev. A 84, 023415 (2011).

[12] S. Martínez-Garaot, M. Palmero, J. G. Muga, and D. GuéryOdelin, Fast driving between arbitrary states of a quantum particle by trap deformation, Phys. Rev. A 94, 063418 (2016).

[13] S. Martínez-Garaot, E. Torrontegui, X. Chen, and J. G. Muga, Shortcuts to adiabaticity in three-level systems using Lie transforms, Phys. Rev. A 89, 053408 (2014).

[14] S. Masuda and K. Nakamura, Fast-forward of adiabatic dynamics in quantum mechanics, Proc. R. Soc. A 466, 1135 (2010).

[15] S. Masuda and K. Nakamura, Acceleration of adiabatic quantum dynamics in electromagnetic fields, Phys. Rev. A 84, 043434 (2011).

[16] J. G. Muga, M. A. Simón, and A. Tobalina, How to drive a Dirac system fast and safe, New J. Phys. 18, 021005 (2016).

[17] V. Mukherjee, S. Montangero, and R. Fazio, Local shortcut to adiabaticity for quantum many-body systems, Phys. Rev. A 93, 062108 (2016). 
[18] D. Sels and A. Polkovnikov, Minimizing irreversible losses in quantum systems by local counterdiabatic driving, Proc. Natl. Acad. Sci. U.S.A. 114, 3909 (2017).

[19] J. Song, Z. Zhang, Y. Xia, X. Sun, and Y. Jiang, Fast coherent manipulation of quantum states in open systems, Opt. Express 24, 21674 (2016).

[20] X. K. Song, F. G. Deng, L. Lamata, and J. G. Muga, Robust state preparation in quantum simulations of Dirac dynamics, Phys. Rev. A 95, 022332 (2017).

[21] K. Takahashi, Unitary deformations of counterdiabatic driving, Phys. Rev. A 91, 042115 (2015).

[22] B. T. Torosov, G. Della Valle, and S. Longhi, NonHermitian shortcut to adiabaticity, Phys. Rev. A 87, 052502 (2013).

[23] E. Torrontegui, S. Martínez-Garaot, and J. G. Muga, Hamiltonian engineering via invariants and dynamical algebra, Phys. Rev. A 89, 043408 (2014).

[24] E. Torrontegui, S. Martínez-Garaot, A. Ruschhaupt, and J. G. Muga, Shortcuts to adiabaticity: Fast-forward approach, Phys. Rev. A 86, 013601 (2012).

[25] G. Vacanti, R. Fazio, S. Montangero, G. M. Palma, M. Paternostro, and V. Vedral, Transitionless quantum driving in open quantum systems, New J. Phys. 16, 053017 (2014).

[26] S. An, D. Lv, A. del Campo, and K. Kim, Shortcuts to adiabaticity by counterdiabatic driving in trapped-ion transport, Nat. Commun. 7, 12999 (2016).

[27] M. G. Bason, M. Viteau, N. Malossi, P. Huillery, E. Arimondo, D. Ciampini, R. Fazio, V. Giovannetti, R. Mannella, and O. Morsch, High-fidelity quantum driving, Nat. Phys. 8, 147 (2011).

[28] J.-F. Schaff, X.-L. Song, P. Capuzzi, P. Vignolo, and G. Labeyrie, Shortcut to adiabaticity for an interacting Bose-Einstein condensate, Europhys. Lett. 93, 23001 (2011).

[29] J.-F. Schaff, X.-L. Song, P. Vignolo, and G. Labeyrie, Fast optimal transition between two equilibrium states, Phys. Rev. A 82, 033430 (2010).

[30] A. Walther, F. Ziesel, T. Ruster, S. T. Dawkins, K. Ott, M. Hettrich, K. Singer, F. Schmidt-Kaler, and U. Poschinger, Controlling Fast Transport of Cold Trapped Ions, Phys. Rev. Lett. 109, 080501 (2012).

[31] T. Wang, Z. Zhang, L. Xiang, Z. Jia, P. Duan, W. Cai, Z. Gong, Z. Zong, M. Wu, J. Wu, L. Sun, Y. Yin, and G. Guo, The experimental realization of high-fidelity 'shortcut-to-adiabaticity' quantum gates in a superconducting xmon qubit, New J. Phys. 20, 065003 (2018).

[32] J. Zhang, J. H. Shim, I. Niemeyer, T. Taniguchi, T. Teraji, H. Abe, S. Onoda, T. Yamamoto, T. Ohshima, J. Isoya, and D. Suter, Experimental Implementation of Assisted Quantum Adiabatic Passage in a Single Spin, Phys. Rev. Lett. 110, 240501 (2013).

[33] J. Deng, Q. H. Wang, Z. Liu, P. Hänggi, and J. Gong, Boosting work characteristics and overall heat engine performance via accelerated adiabatic control: Quantum and classical, Phys. Rev. E 88, 062122 (2013).

[34] C. Jarzynski, Generating shortcuts to adiabaticity in quantum and classical dynamics, Phys. Rev. A 88, 040101 (2013).
[35] C. Jarzynski, S. Deffner, A. Patra, and Y. Subasi, Fast forward to the classical adiabatic invariant, Phys. Rev. E 95, 032122 (2017).

[36] M. Okuyama and K. Takahashi, From Classical Nonlinear Integrable Systems to Quantum Shortcuts to Adiabaticity, Phys. Rev. Lett. 117, 070401 (2016).

[37] A. Patra and C. Jarzynski, Classical and quantum shortcuts to adiabaticity in a tilted piston, J. Phys. Chem. B 121, 3403 (2017).

[38] E. Torrontegui, I. Lizuain, S. González-Resines, A. Tobalina, A Ruschhaupt, R. Kosloff, and J. G. Muga, Energy consumption for shortcuts to adiabaticity, Phys. Rev. A 96, 022133 (2017).

[39] M. Chupeau, B. Besga, D. Guéry-Odelin, E. Trizac, A. Petrosyan, and S. Ciliberto, Thermal bath engineering for swift equilibration, Phys. Rev. E 98, 010104 (2018).

[40] G. Li, H. T. Quan, and Z. C. Tu, Shortcuts to isothermality and nonequilibrium work relations, Phys. Rev. E 96, 012144 (2017).

[41] I. A. Martínez, A. Petrosyan, D. Guéry-Odelin, E. Trizac, and S. Ciliberto, Engineered swift equilibration of a Brownian particle, Nat. Phys. 12, 843 (2016).

[42] A. Patra and C. Jarzynski, Shortcuts to adiabaticity using flow fields, New J. Phys. 19, 125009 (2017).

[43] Z. C. Tu, Stochastic heat engine with the consideration of inertial effects and shortcuts to adiabaticity, Phys. Rev. E 89, 052148 (2014).

[44] D. J. Griffiths, Introduction to Quantum Mechanics, 2nd ed. (Prentice Hall, Upper Saddle River, 2004).

[45] S. Masuda, Acceleration of adiabatic transport of interacting particles and rapid manipulations of a dilute Bose gas in the ground state, Phys. Rev. A 86, 063624 (2012).

[46] S. Masuda, K. Nakamura, and A. del Campo, High-Fidelity Rapid Ground-State Loading of an Ultracold Gas into an Optical Lattice, Phys. Rev. Lett. 113, 063003 (2014).

[47] S. Masuda, K. Nakamura, and M. Nakahara, Fast-forward scaling theory for phase imprinting on a BEC: Creation of a wave packet with uniform momentum density and loading to Bloch states without disturbance, New J. Phys. 20, 025008 (2018).

[48] S. Masuda and S. A. Rice, Rapid coherent control of population transfer in lattice systems, Phys. Rev. A 89, 033621 (2014).

[49] E. Torrontegui, X. Chen, M. Modugno, S. Schmidt, A. Ruschhaupt, and J. G. Muga, Fast transport of Bose-Einstein condensates, New J. Phys. 14, 013031 (2012).

[50] A. Kiely, J. P. L. McGuinness, J. G. Muga, and A. Rauschhaupt, Fast and stable manipulation of a charged particle in a Penning trap, J. Phys. B 48, 075503 (2015).

[51] S. Masuda and S. A. Rice, Rotation of the orientation of the wave function distribution of a charged particle and its utilization, J. Phys. Chem. B 119, 11079 (2015).

[52] S. Masuda and S. A. Rice, Fast-forward assisted stirap, J. Phys. Chem. A 119, 3479 (2015).

[53] See Supplemental Material at http://link.aps.org/supplemental/ 10.1103/PhysRevResearch.3.013087 for movies showing the evolving profile of $p_{k}(t)$ both without and with the fast-forward potential, using the same parameters as in Fig. 2, except $\hbar=1$ and $\psi_{0}=|35\rangle$.

[54] H. Goldstein, Classical Mechanics, 2nd ed. (Addison-Wesley, Reading, 1980).

[55] R. G. Littlejohn, The Van Vleck formula, Maslov theory, and phase space geometry, J. Stat. Phys. 68, 7 (1992). 